\title{
La desnutrición en la niñez argentina en los primeros años del siglo XXI: un abordaje cuantitativo
}

\author{
Child malnutrition in Argentina in the first part of the \\ century: a quantitative approach
}

Fernando Longhi ${ }^{1}$, Alicia Gómez², María Elisa Zapata ${ }^{3}$, Pablo Paolasso4, Fernanda Olmos 5 , Silvina Ramos Margarido ${ }^{6}$

${ }^{1}$ Doctor en Ciencias Sociales. Investigador adjunto, Consejo Nacional de Investigaciones Científicas y Técnicas, Instituto Superior de Estudios Sociales, Universidad Nacional de Tucumán, Argentina. $\bowtie$ (iD

2Licenciada en Sociología. Docente, Maestría en Demografía Social, Universidad Nacional de Luján, Argentina. $\triangle$ (iD)

${ }^{3}$ Magíster en Nutrición Humana y Calidad de los Alimentos. Investigadora adjunta, Centro de Estudios sobre Nutrición Infantil, Argentina. $\square$ (iD)

${ }^{4}$ Doctor en Geografía. Investigador independiente, Consejo Nacional de Investigaciones Científicas y Técnicas, Instituto Superior de Estudios Sociales, Universidad Nacional de Tucumán, Argentina. $\bowtie$ (iD

${ }^{5}$ Magíster en Demografía Social. Docente, Maestría en Demografía Social, Universidad Nacional de Luján, Argentina. $\bowtie$ (iD)

${ }^{6}$ Licenciada en Sociología. Docente, Universidad de Buenos Aires, Argentina. $\triangle$ iD
RESUMEN La desnutrición en la niñez conforma un importante problema de salud pública en Argentina, ya sea como causa básica de mortalidad/morbilidad o asociada a distintas patologías que inciden sobre la población infantil. Sin embargo, poco se conoce sobre su magnitud, tendencias y su distribución espacial. Este artículo procuró detectar estas situaciones considerando diferentes escalas geográficas. Se propuso un abordaje cuantitativo mediante la sistematización de estadísticas vitales (mortalidad), egresos hospitalarios (morbilidad), y de bajo peso al nacimiento (natalidad), sobre una población objetivo de 0 a 4 años de edad. Las fuentes de información utilizadas fueron las estadísticas de mortalidad (1999-2013), estadísticas de egresos hospitalarios (2000, 2005-2011) y estadísticas de nacidos vivos (1999-2012) provistos por la Dirección de Estadísticas e Información de Salud (DEIS) del Ministerio de Salud de la Nación. Los resultados advierten comportamientos diferenciados según la escala considerada. Como conclusión, se destaca la necesidad de integrar las vertientes de información analizadas para brindar un panorama más general sobre un problema que, si bien tiende a descender, alcanza magnitudes altas en las zonas más vulnerables. Ciertas áreas del norte presentan las peores condiciones y precisan un abordaje inmediato en materia de pobreza y salud infantil.

PALABRAS CLAVES Desnutrición; Trastornos de la Nutrición del Niño; Geografía Médica; Pobreza; Argentina.

ABSTRACT Child malnutrition is an important public health problem in Argentina, both as a primary cause of mortality/morbidity and associated with different pathologies that affect children. However, little is known about its magnitude, trends and spatial distribution. This article seeks to detect such situations considering different geographic scales. A quantitative approach was applied, systematizing vital statistics (mortality), hospital discharges (morbidity), and low birth weight (natality) in children 0-4 years of age. Accordingly, the information sources used were mortality statistics (1999-2013), hospital discharge statistical records (2000, 2005-2011) and live birth statistics (19992012) provided by the Office of Statistics and Health Information (DEIS) [Dirección de Estadísticas e Información de Salud] of the National Ministry of Health. The results show differences according to the scale considered. The conclusions highlight the necessity of integrating the different sources of information analyzed in order to provide a more general overview of a problem that, albeit in decline, still registers high magnitudes in the most vulnerable areas. In this way, certain areas of northern Argentina evidence worse conditions, requiring immediate attention be paid to issues of poverty and child health.

KEY WORDS Malnutrition; Infant Nutrition Disorders; Medical Geography; Poverty; Argentina. 


\section{INTRODUCCIÓN}

A fines de la primera década del siglo XXI los países de América Latina, en general, y Argentina, en particular, habían registrado grandes avances en sus condiciones de vida, tomando como referencia para la comparación la década de 1990. Este proceso reconoció el avance de condiciones políticas y económicas que favorecieron el reposicionamiento del Estado en la programación e implementación de políticas públicas ${ }^{(1)}$. Sin embargo, en el trienio 2011-2013, recientes investigaciones han registrado cierto estancamiento y/o retrocesos en algunas de las dimensiones fundamentales del bienestar social, entre las que se encuentra la situación nutricional de los niños ${ }^{(2)}$.

El estado de nutrición, como proceso, está condicionado por distintos factores. Es conocido que una adecuada nutrición, durante la etapa fetal y en los primeros años de vida, es fundamental para el crecimiento y desarrollo del niño; las consecuencias de una inadecuada nutrición se reflejan en el corto, mediano y largo plazo en el individuo y en la sociedad a la que pertenece. La deficiencia en su implementación, sobre todo en los primeros años de vida, tiene graves consecuencias en distintos aspectos tanto para el individuo como para la sociedad a la que pertenece. Según el Programa Mundial de Alimentos de Naciones Unidas, en América Latina la desnutrición afecta a casi 9 millones de niños menores de cinco años $(16 \%)^{(3)}$. Se agregan a esta cifra 9 millones más de niños con alto riesgo de desnutrirse debido a las condiciones de pobreza en las que viven. De todos estos, una buena proporción se localizan en países como Guatemala, Bolivia y Honduras.

En Argentina, la prevalencia de desnutrición sería relativamente baja si se compara con el contexto latinoamericano. Sin embargo, ya a inicios del siglo XXI, la Food and Agriculture Organization (FAO) destacaba que "si bien en Argentina la disponibilidad de alimentos es suficiente y aun excedente para cubrir las necesidades energéticas por persona y por día, parte de la población tiene un acceso insuficiente a los alimentos"(2). Diversos estudios ${ }^{(4,5)}$ describen que las formas de desnutrición prevalentes en Argentina antes de la década de 2000 eran el déficit de talla y la desnutrición crónica, siendo el norte la región más afectada ${ }^{(6)}$. Por otro lado, los resultados de la Encuesta Nacional de Nutrición y Salud (ENNYS) realizada en 2004-2005 consolidaban las afirmaciones anteriores, revelando una magnitud sorprendente de niños afectados con anemia (Ilamada también desnutrición oculta) la cual alcanzaba en Chaco, por ejemplo, al $36 \%$ de los niños de 6 a 72 meses, siendo el valor nacional de $16,5 \%^{(7)}$. La cara más dura de la desnutrición, es decir el bajo peso para la edad, alcanzaba en el nordeste argentino al 3\% de los niños de 6 a 60 meses de edad. Todas estas investigaciones, de alguna manera, ponen énfasis en el problema nutricional que caracteriza a la niñez argentina.

La crisis de 2001-2002 habría repercutido de forma negativa en la situación nutricional del país, puesto que la devaluación monetaria, el resurgimiento de la inflación, el deterioro en los indicadores de empleo y la desigualdad en la distribución de los ingresos crearon las condiciones para un fuerte empeoramiento de la situación alimentaria, provocando una grave crisis en el acceso a los alimentos de gran parte de la población a comienzos del 2002. Pudo así observarse que los problemas nutricionales estaban lejos de ser inexistentes y adquirían, en los albores del siglo XXI, magnitudes elevadas para Argentina.

Esta situación habría sido gradualmente revertida desde el año 2003 a partir de una intensiva aplicación de programas destinados a su reducción (Plan Nacional de Seguridad Alimentaria, por ejemplo) y el mejoramiento de las condiciones económicas y sociales del país. No obstante, había un desconocimiento acerca de la magnitud de la incidencia de la desnutrición en la niñez y el efecto -atendiendo a las desigualdades regionales existentes- de la aplicación de dichas políticas.

En este contexto, cabe señalar que Argentina presentaba, a fines de 2014, los 
siguientes aspectos relevantes que resultan de interés en la consideración de la temática:

a. Gran variedad de políticas y programas sociales, muchas veces inconexas entre sí, tendientes a satisfacer las necesidades sociales de amplios sectores de la población que habían quedado en situación de vulnerabilidad como resultado de las políticas neoliberales de la década de 1990.

b. Desconocimiento respecto de la evolución anual y de las tendencias de los índices de desnutrición, y falta de información sistemática y continua sobre la situación de la desnutrición infantil.

c. Desigual acceso a la salud, lo cual se traduce en la existencia de profundas desigualdades socioeconómicas, culturales y ambientales.

En este contexto, cabe preguntarse: ¿cuál fue la incidencia del problema de la desnutrición de niños en los últimos años?, ¿qué tendencias presenta?, ¿cómo varió su distribución espacial y cómo evolucionó en el tiempo? Estos interrogantes propiciaron el desarrollo de herramientas conceptuales y metodológicas específicas que permitieron describir y analizar adecuadamente el problema, así como poder conjeturar cierto tipo de respuestas.

Sobre la base del contexto que hemos reseñado y las preguntas que surgen, planteamos los siguientes objetivos:

a. Describir el patrón de evolución de las defunciones por desnutrición de los menores de cinco años, su incidencia y su distribución espacial en el período 1999-2013, considerando la escala nacional, regional, provincial y departamental.

b.Explorar el patrón de evolución de los egresos hospitalarios por desnutrición y causas asociadas de los menores de cinco años, su incidencia y distribución espacial durante el período 2000-2010.

c. Analizar los caracteres de los niños con bajo peso al nacer con la finalidad de definir un indicador de desnutrición que permita complementar el análisis previo sobre la desnutrición infantil.

\section{Fundamentos teórico-conceptuales}

La desnutrición es el resultado fisiopatológico de una ingesta de alimentos insuficientes. Dicha insuficiencia se caracteriza por su continuidad temporal y puede responder también a procesos en los que la capacidad de absorción está disminuida o a defectos metabólicos en los que existe una inadecuada utilización biológica de los nutrientes consumidos ${ }^{(8)}$. Actualmente, se considera que los conceptos de nutrición/desnutrición están atravesados por tres dimensiones: la biológica, que entiende la nutrición como un proceso indispensable para el mantenimiento de la vida; la social, en la que intervienen factores culturales tales como la religión, la educación y los hábitos alimentarios, así como diferentes factores económicos; y la ambiental, en la que se menciona la importancia de la sustentabilidad de la producción de alimentos y la utilización de cultivos como fuente de energía, en tanto medidas que no solo contribuyen a la nutrición de las poblaciones sino que también permiten la conservación del medio ambiente ${ }^{(9)}$.

Los determinantes de la desnutrición pueden clasificarse en inmediatos, subyacentes y básicos (o estructurales según nuestra propuesta ${ }^{(10)}$. Entre los determinantes inmediatos se encuentran las dietas insuficientes (en cantidad y calidad) y algunas enfermedades reincidentes, entre las que se destacan las infecciones diarreicas, respiratorias y las parasitosis. A esta condición se suma la escasa inmunización.

Dentro de los determinantes subyacentes se encuentran la inseguridad alimentaria, la falta de asistencia médica, inadecuados servicios de salud, agua y saneamientos unidos a malas condiciones higiénicas, entre otros. Sin embargo, el determinante básico/estructural principal es la pobreza(10,11) (Figura 1).

Un concepto intermediario de gran relevancia que incide sobre el advenimiento y desarrollo de la desnutrición en los niños, es el de estrategias de supervivencia para el cuidado infantil, entendidas como ciertas conductas y habilidades con las cuales la familia enfrenta ciertas condiciones macro y micro institucionales que afectarían su salud. 


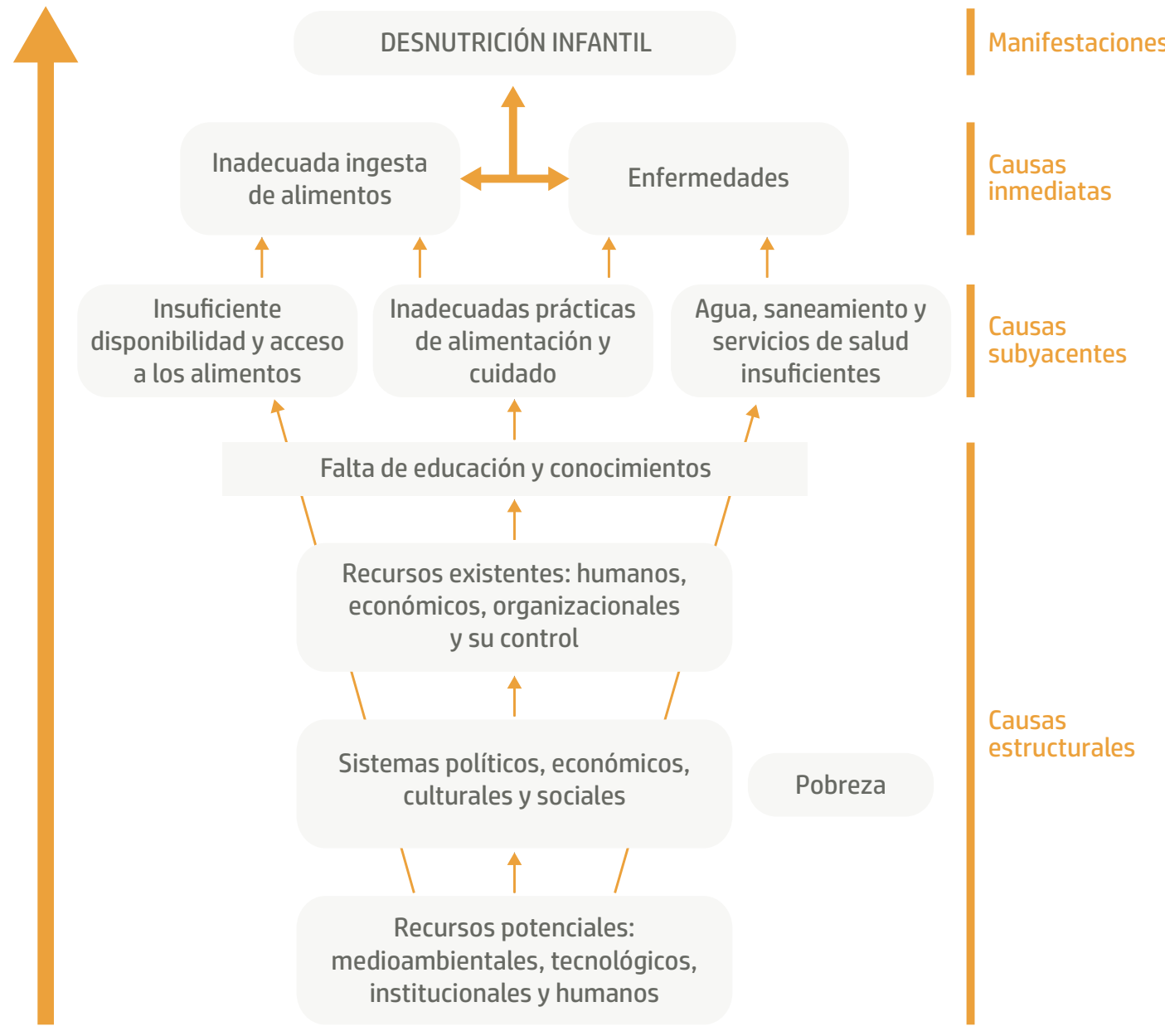

Figura 1. Modelo causal de la desnutrición infantil.

Fuente: Elaboración propia a partir de Unicef ${ }^{(11)}$.

En el seno de la familia surgen habilidades respecto de cómo satisfacer las necesidades de manutención y reproducción de los miembros con los recursos disponibles. Tales conductas tienden a asegurar la reproducción biológica de la familia, preservar la vida y efectuar todas aquellas prácticas, económicas y no económicas, indispensables para la obtención de los medios de subsistencia y para la socialización de la progenitura ${ }^{(12)}$. Una de estas estrategias se vincula con la inclusión de la familia en una red social estructurada y continua. Bronfman ${ }^{(13)}$ destaca el papel de estas redes, sobre todo en familias pobres, ya que considera que son frecuentemente la única posibilidad de ayuda con que pueden contar y el único soporte para aligerar las pesadas cargas de la vida cotidiana. Si bien la presencia de estas estrategias no participa directamente en la ocurrencia de muertes, evita muchas veces que accidentes y enfermedades tengan desenlaces fatales.

Según diferentes evidencias, existe una fuerte relación entre la pobreza -en sus distintas formas y manifestaciones- y la desnutrición. Esta relación no determina la muerte, 
sino que la condiciona de manera importante $y$, aunque el circuito no termine necesariamente en una defunción, las secuelas dejadas principalmente en los niños pueden traer como consecuencia serias limitaciones tanto en sus aspectos físicos, como psíquicos, cognitivos y afectivos las que, a su vez, pueden incidir sobre los niveles de pobreza.

Por otro lado, aun cuando la desnutrición no hubiese provocado la muerte, genera una serie de efectos o consecuencias en los individuos en su adultez que conciernen a su estado físico lo cual incluye: baja estatura, una menor masa muscular, capacidad limitada para el trabajo y mayor riesgo de obesidad y de enfermedades crónicas. Asimismo, la evidencia sobre los efectos de la desnutrición temprana en el rendimiento escolar y el desempeño intelectual es sólida también ${ }^{(14)}$. Entre los efectos ya conocidos de la desnutrición temprana se puede incluir el ingreso tardío al primer grado, bajo grado de escolaridad y limitaciones en lectura y vocabulario; vale decir que estos efectos se mantienen después de controlarlos por factores de confusión. La escolaridad y las capacidades derivadas de la desnutrición condicionan la trayectoria ocupacional y los ingresos económicos, aun en áreas rurales tradicionales ${ }^{(15)}$.

Entre 1969 y 1977, el Instituto de Nutrición de Centroamérica y Panamá (INCAP) realizó un estudio longitudinal en el oriente de Guatemala ${ }^{(16)}$, que consistió en un ensayo comunitario aleatorizado en el que los pobladores de dos aldeas, una grande y otra pequeña, recibieron un suplemento nutricional denominado "Atole" y los pobladores de otras dos aldeas similares recibieron una bebida de control Ilamada "Fresco". Atole era una bebida tipo papilla con aporte de micronutrientes, calorías y proteínas, mientras que Fresco era una bebida que aportaba micronutrientes, baja en calorías, y con un aporte insignificante de proteínas. Desde el punto de vista biológico, el experimento nutricional resultó eficaz para mejorar la ingesta dietaria y el crecimiento en la longitud de los niños, si bien el impacto sobre el crecimiento solo pudo corroborarse en los primeros 3 años de vida. Entre 1988 y 1989 se realizó el primer estudio de seguimiento de los participantes, que arribó a la conclusión que una nutrición mejorada en los primeros años de vida tiene efectos importantes en el capital humano, según los parámetros de tamaño corporal, capacidad de trabajo y funcionamiento intelectual. Sin embargo, debido a la corta edad de los participantes, el estudio no pudo vincular la intervención nutricional con la productividad económica ${ }^{(15)}$.

En este sentido, Martínez y Fernández ${ }^{(17)}$ sostienen que la desnutrición infantil presenta una serie de consecuencias negativas en distintos ámbitos: se destacan los impactos en morbimortalidad, educación y productividad, constituyéndose en uno de los principales mecanismos de transmisión intergeneracional de la pobreza y la desigualdad. Así, la desnutrición en gestantes aumenta el riesgo de bajo peso al nacer e incrementa, a su vez, el riesgo de muerte neonatal. Los bebés que nacen con un peso de 2.000 a 2.499 gramos enfrentan un riesgo de muerte neonatal que cuadruplica el de aquellos que pesan entre 2.500 y 2.999 gramos, y es 10 a 14 veces superior respecto de los que pesan al nacer entre 3.000 y 3.499 gramos. En distintos estudios se observa que la desnutrición es el mayor contribuyente de la mortalidad infantil y de la niñez en edad preescolar ${ }^{(18)}$ (entre el $50 \%$ y el $60 \%$ ), mientras el porcentaje de casos de morbilidad atribuible a la desnutrición es del $61 \%$ para la diarrea, el $57 \%$ para la malaria, el $53 \%$ para la neumonía y el $45 \%$ para el sarampión, e incrementa significativamente el riesgo de que en la edad adulta se desarrollen patologías crónicas, tales como enfermedades coronarias, hipertensión y diabetes y enfermedades transmisibles como la tuberculosis ${ }^{(17)}$.

Se entiende así que la pobreza, determinante básico de la desnutrición en la niñez, alude siempre a la situación en la que se encuentran las familias. Dicha situación no es la suma o el agregado más o menos independiente de dimensiones o aspectos parciales, sino el cuadro situacional estructural en el que se hallan, producto de su particular inserción en la estructura socioproductiva, siendo esta la que determina la posibilidad 
de acceder o no a ciertos bienes y servicios ${ }^{(19)}$. Entendemos que en este contexto nace y se desarrolla la desnutrición en la niñez.

\section{FUENTES Y MÉTODOS}

Esta propuesta se enmarca temporalmente en los años comprendidos entre 1999 y 2013. La población objeto de estudio está constituida por la niñez argentina con edades entre cero y cuatro años. Según el Censo Nacional de Población, Hogares y Viviendas 2010, del total de la población argentina (40 millones), un poco más de 3 millones de personas, tenían entre cero y cuatro años. Este grupo poblacional representaba el $8,3 \%$ del total.

El estudio de la desnutrición en la niñez argentina implicó abordar un problema de difícil captación en líneas generales y, especialmente, cuando solo se visibiliza su expresión extrema. En este sentido, la disponibilidad y características de las fuentes de información conformó un determinante sustancial para la generación de las herramientas metodológicas y los resultados hallados. De esta manera, la información seleccionada para dar cuenta del problema debe ser considerada, teniendo presente las limitaciones de la calidad de los datos disponibles.

Las fuentes de información utilizadas fueron las estadísticas de mortalidad (19992013), estadísticas de egresos hospitalarios (2000, 2005-2011) y estadísticas de nacidos vivos (1999-2012) provistos por la Dirección de Estadísticas e Información de Salud (DEIS) del Ministerio de Salud de la Nación. De las tres fuentes mencionadas, la información que presenta menor solidez es la referida a egresos hospitalarios, la cual remite solo a los egresos de los hospitales públicos y su consolidación estadística está en proceso. Por tales motivos, aún presenta deficiencias en su nivel de cobertura y completitud en el período seleccionado. Se usaron también los Censos Nacionales de Población, Hogares y Viviendas 2001 y 2010, con los cuales se estimó la población media.

A partir de las estadísticas de mortalidad, se construyó la tasa de mortalidad por desnutrición en la niñez (TMDN), un indicador que relaciona la cantidad de muertes registradas por dicha patología en menores de cinco años, respecto de la población menor de cinco años del área seleccionada, expresada cada mil menores. La categoría desnutrición y otras anemias nutricionales está integrada por las siguientes patologías de la CIE 10: anemias por falta de hierro (D50), anemia por deficiencia de vitamina B12 (D51), anemia por deficiencia de folatos (D52), otras anemias nutricionales (D53), Kwashiorkor (E40), marasmo nutricional (E41), Kwashiorkor marasmático (E42), desnutrición proteinocalórica (E43-E46), deficiencia de vitamina A (E50), deficiencia de tiamina (E51), pelagra (E52), deficiencia de otras vitaminas del grupo B (E53), deficiencia de ácido ascórbico (E54), deficiencia de vitamina D (E55), otras deficiencias de vitaminas (E56), deficiencia dietética de calcio (E58), deficiencia dietética de selenio (E59), deficiencia dietética de zinc (E60), deficiencia de otros elementos nutricionales (E61), otras deficiencias nutricionales (E63) y secuelas de la desnutrición y de otras deficiencias nutricionales (E64).

Este indicador reviste un carácter extremo al considerar solo la defunción por desnutrición, lo cual soslaya buena parte del problema. Es por esta razón que se apeló a la utilización de otras fuentes no tradicionales en el análisis de la desnutrición.

Se consolidaron las estadísticas de egresos hospitalarios, a pesar de no contar con una serie de datos tan completa, a fin de lograr un punto de referencia del posible alcance del problema. En su tratamiento se adoptaron los mismos criterios utilizados para el procesamiento de las estadísticas vitales, obteniendo así los aspectos vinculados con la morbilidad.

Una tercera vertiente de aproximación implicó la generación de un indicador que considere el bajo peso al nacimiento (menos de 2.500 gramos) a término, proveniente de madres con educación insuficiente a partir de las estadísticas de nacimientos. En este caso, se calculó la proporción que representa la cantidad de nacidos vivos provenientes de gestaciones iguales o superiores a las treinta 
y siete semanas, provenientes de madres con nivel educativo igual a primario incompleto, relacionados con el total de nacidos vivos para cada área geográfica. El bajo peso al nacer, cuando es adecuadamente registrado, constituye otro valioso indicador nutricional, ya que resume los cuidados prenatales que la madre ha recibido durante la gestación. Este indicador puede asociarse a compromiso nutricional de la madre -aunque, para que se manifieste, debe ser muy severo- $y$, generalmente, expresa un control gestacional tardío y deficiente por parte de la madre ${ }^{(20)}$. Es preciso destacar que esto constituye la manifestación extrema de un problema aun mayor dado que, en muchos casos, existe un elevado nivel de subregistro o la calidad de la información es defectuosa. Ejemplos de esto lo constituyen los casos en los que existiendo una desnutrición de base, la muerte no fue codificada como tal.

Pese a las limitaciones de la calidad del registro, el problema de la muerte por desnutrición adquiere una relevancia importante dentro de la mortalidad general y, específicamente, en la mortalidad infantil. Shanghvi y Murray ${ }^{(21)}$ manifiestan que la desnutrición, aun en sus formas más benignas, puede estar relacionada hasta con el $56 \%$ de toda la mortalidad infantil.

Los indicadores derivados de estas tres vertientes de análisis pueden ser interpretados en un registro espacial, según su distribución, reconociendo tres entidades de análisis: seis regiones geográficas, las veintitrés provincias y los quinientos once departamentos o partidos que conforman el territorio argentino. La construcción de la tasa difiere según la escala considerada: en el caso nacional, regional y provincial se calculó una tasa cuyo denominador consistió en la población de cero a cuatro años, obtenida de las proyecciones demográficas empalmadas con la serie 2010 del Programa de Análisis Demográfico del Instituto Nacional de Estadística y Censos (INDEC). Finalmente, en la escala departamental, se consideró la proporción de muertes de cada caso respecto al total de defunciones, egresos o nacidos vivos según el caso (esta distinción fue necesaria debido a que no disponemos de proyecciones de población a nivel departamental en estos tramos de edad). Para la elaboración cartográfica, se clasificó en todos los casos la variable en cuatro categorías, por lo que cada una representa el $25 \%$ de los datos de la distribución (cuartiles); siendo la primera categoría la que representa las jurisdicciones con la inexistencia de casos de la variable analizada. Asimismo, se calcularon los umbrales de las categorías incluyendo los datos de las tres distribuciones espaciales consideradas, esto posibilitó mantener los mismos umbrales en los distintos mapas y permitió la comparación en la evolución de dichas distribuciones.

Para dar cuenta de las tendencias del problema en la década bajo estudio, se realizó un análisis diacrónico anual a escala nacional, regional y provincial. Para la escala departamental se realizó un agrupamiento quinquenal de los datos, esto responde a la diversidad en la cantidad de casos ocurridos en los distintos departamentos. De esta manera se obtiene una tendencia que evita las oscilaciones anuales bruscas en el indicador.

Finalmente, cabe mencionar que se aplicó un diseño observacional, descriptivo y transversal. Esta estrategia requirió la recopilación sistemática de datos y la elaboración de indicadores a partir de información derivada de las estadísticas de mortalidad, morbilidad y nacimientos, asociados a la desnutrición en la niñez para diferentes escalas geográficas en diferentes periodos de la primera década del siglo XXI. En términos generales, los lapsos de análisis abarcan los períodos 2000-2010 y 1999-2013 según las características de cada una de las fuentes.

\section{LA DESNUTRICIÓN INFANTIL ARGENTINA SEGÚN DIFERENTES ESCALAS DE ANÁLISIS}

\section{La escala nacional}

En la Figura 2 se observa que la distribución de la mortalidad de los menores de 5 años por causa básica de desnutrición y anemias nutricionales muestra en el 
transcurso de la década un sostenido descenso luego de la crisis de los años 2002 y 2003, cuando alcanzó un pico de 0,07 por mil. A partir del año 2003, se observa un descenso sostenido hasta alcanzar en el año 2013 una tasa de 0,01 por mil. La curva de los egresos hospitalarios, en tanto, presenta valores notoriamente más elevados que la mortalidad. En este caso, el registro supera entre 19 y 45 veces el valor de la mortalidad (según el año que se considere), con una tendencia al descenso, aunque menos pronunciada que el de la mortalidad.

La proporción de nacimientos de bajo peso (menos de 2.500 gramos) a término provenientes de madres con educación crítica presenta también un notorio descenso, con registros del orden del $4 \%$ en 1999 a un $1,5 \%$ en 2012. Se observa un quiebre en la tendencia alrededor del año 2007, la cual vuelve a retomar luego de ese año un recorrido descendente.

Resulta evidente la importante brecha que existe entre las incidencias de cada uno de los indicadores, lo cual provee un panorama de la amplitud potencial del problema de la desnutrición, magnitud que resulta desconocida tanto en los ámbitos académicos como políticos actuales.

Se puede afirmar, entonces, que aun cuando pueda existir algún nivel de subregistro en las defunciones ocurridas, estrictamente por desnutrición, las otras variables que también registran el fenómeno muestran la misma tendencia. De esta manera, sin Conocer los valores exactos, debido a las limitaciones de las fuentes, se observa una clara tendencia descendente del problema. Es posible inferir además su magnitud y reconocer, a pesar del descenso, la importante incidencia que genera su prevalencia en distintos aspectos de la salud infantil, como así también la probable vigencia de la problemática en sus manifestaciones menos extremas.

\section{La escala regional}

Existe una clara diferenciación regional tanto en las magnitudes como en las

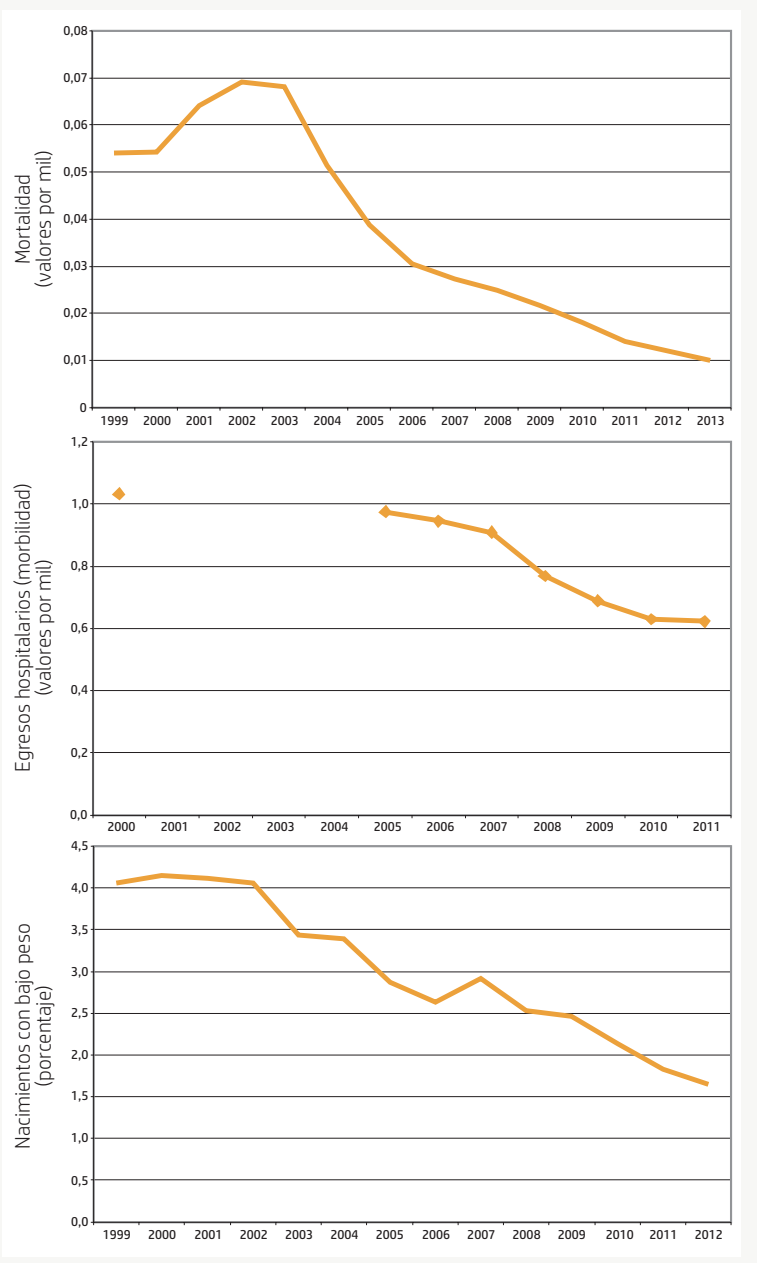

Figura 2. Tasa de mortalidad de menores de cinco años (1999-2013), tasa de egresos hospitalarios (20002011), según desnutrición y anemias y proporción de nacimientos de bajo peso a término de madres con educación insuficiente (1999-2012). Argentina.

Fuente: Elaboración propia con base en datos del Programa Nacional de Estadísticas de Salud, Dirección de Estadísticas e Información de Salud, Ministerio de Salud de la Nación.

tendencias que alcanza la desnutrición en la niñez según las diferentes vertientes aquí analizadas (Figura 3). En todos los casos, el nordeste argentino y el noroeste argentino son las regiones que presentan las peores condiciones, aunque los comportamientos de cada una de las manifestaciones de la desnutrición son muy disímiles.

Mientras que la mortalidad ya había sido controlada durante la década de 1990 en buena parte del país, en el norte constituía todavía un problema no resuelto. Hubo allí 

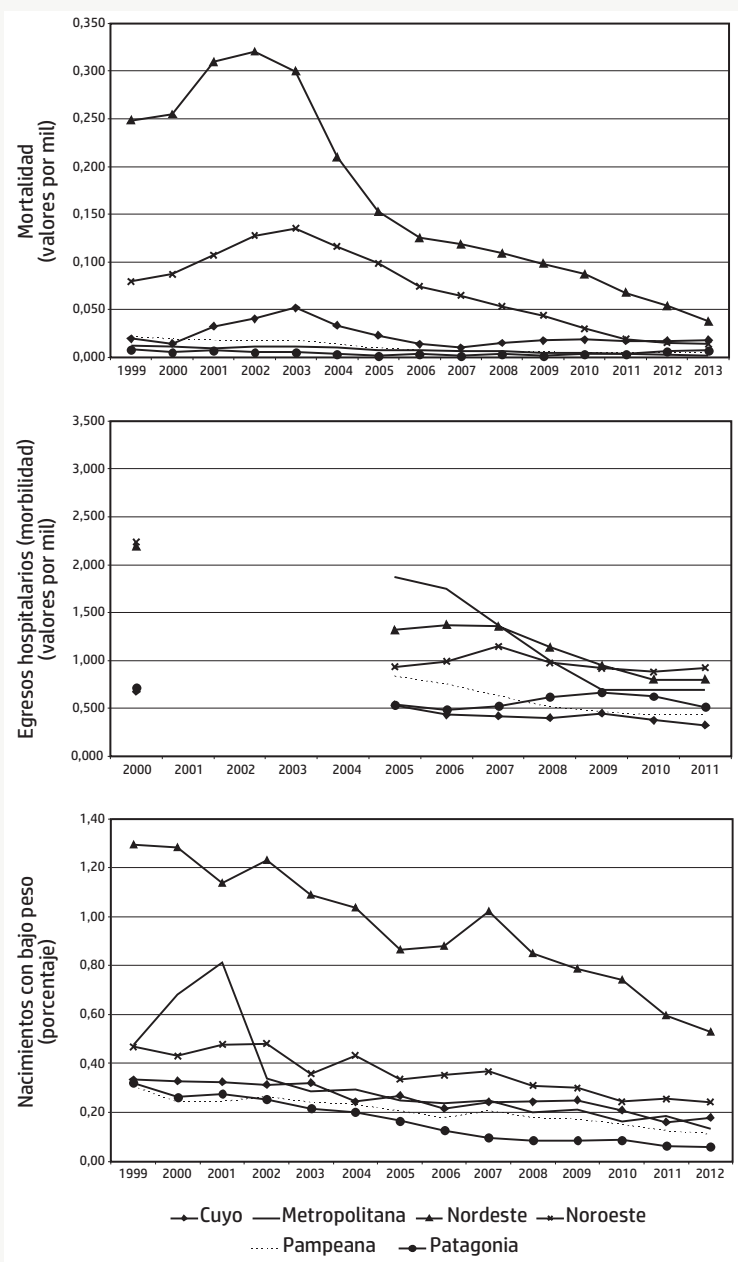

Figura 3. Tasa de mortalidad (1999-2013), egresos hospitalarios (2000-2011) de menores de cinco años según desnutrición y otras anemias nutricionales, y proporción de bajo peso al nacer según edad gestacional a término y baja educación de las madres (1999-2012), por regiones.

Fuente: Elaboración propia con base en datos del Programa Nacional de Estadísticas de Salud, Dirección de Estadísticas e Información de Salud, Ministerio de Salud de la Nación.

avances significativos durante la primera década del presente siglo, sobre todo luego de la crisis que atravesó el país en 2001. Hacia 2013, aun cuando la región nordeste presentaba una brecha importante con respecto al promedio nacional, podía observarse una tendencia a la convergencia en los niveles regionales.

La morbilidad presenta un comportamiento mucho más estable en su tendencia. Asimismo, la diferenciación regional observada en la mortalidad es aún más notoria en este caso. El valor absoluto de los egresos por desnutrición en el periodo alcanzó a 15.684 menores de cinco años, lo cual representa el $0,69 \%$ de los egresos hospitalarios ocurridos de niños en ese tramo de edad. Esta vertiente del problema que buscamos caracterizar muestra un aspecto que permanece oculto en el indicador tradicional analizado, ya que en el Área Metropolitana de Buenos Aires alcanzó, sobre mediados del periodo, los valores más elevados, descendiendo a partir de entonces para ubicarse detrás de la región del noroeste y nordeste como la tercera región con mayores tasas de egreso por desnutrición del país. La vertiente del bajo peso al nacer ayuda a comprender mejor cuáles son las magnitudes de las diferencias regionales $y$, aunque también evidenció una tendencia descendente al igual que los otros aspectos analizados, puede verse claramente que la región del noroeste y nordeste registraron los valores más altos del país.

\section{La escala provincial}

La Figura 4 (mortalidad en la niñez), la Figura 5 (egresos hospitalarios) y la Figura 6 (bajo peso) muestran el comportamiento de cada una de las vertientes según provincias. En el primer caso, es notorio un comportamiento muy diferenciado de la variable, con provincias en las que se advierte prácticamente la inexistencia del problema, tales como San Luis, La Pampa, Tierra del Fuego, Santa Cruz, Río Negro, Chubut, Neuquén y la Ciudad Autónoma de Buenos Aires. Por el contrario, los valores más altos de la tasa se localizan en las provincias la región del nordeste y noroeste, a las que se agrega San Juan, en el caso cuyano. Estas parten de valores muy elevados, en su mayoría, y tendieron al descenso, con excepción de San Juan y Santiago del Estero, provincias en las que se observa un paulatino empeoramiento de la tasa desde el año 2007 en adelante.

Considerando los egresos hospitalarios, la percepción obtenida del análisis de la 
Cuyo

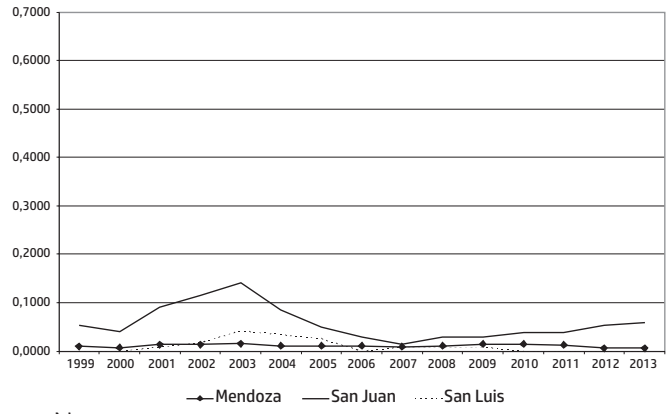

Noroeste

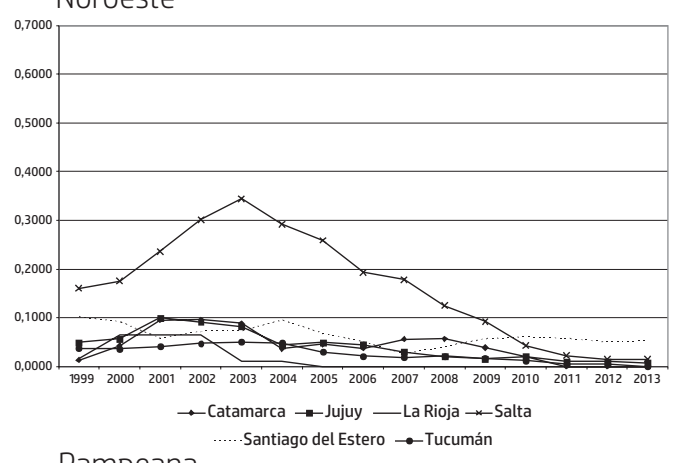

Pampeana

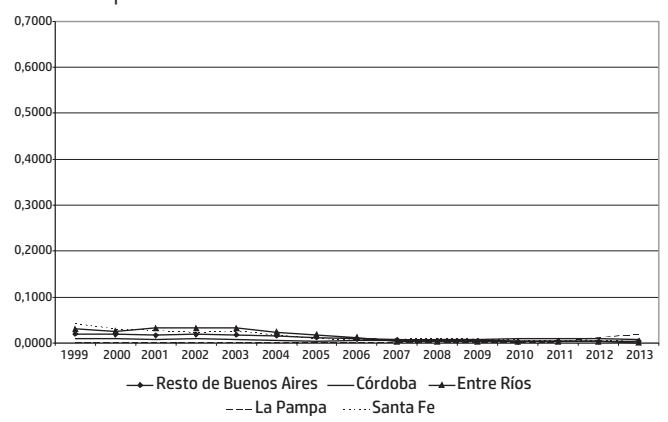

Metropolitana

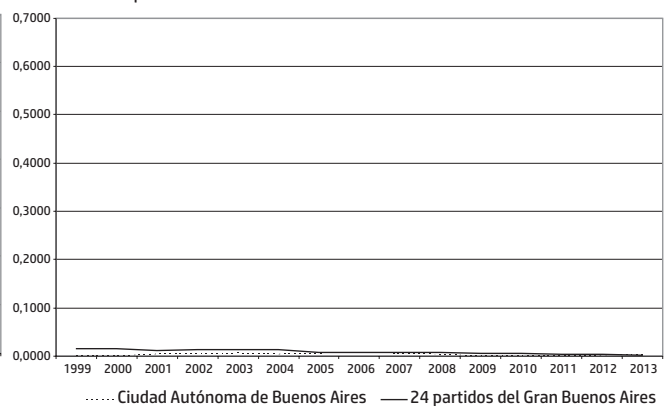
Nordeste

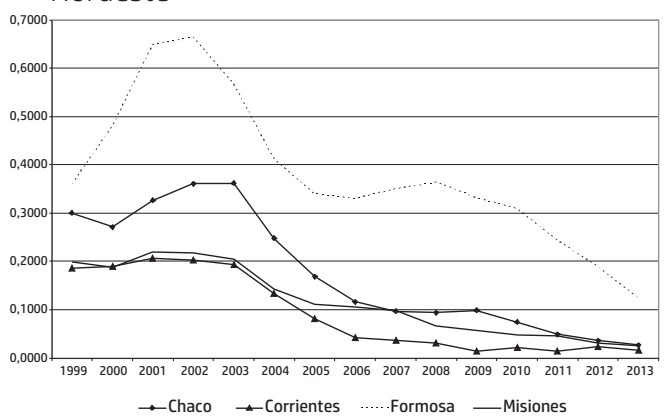

Patagonia

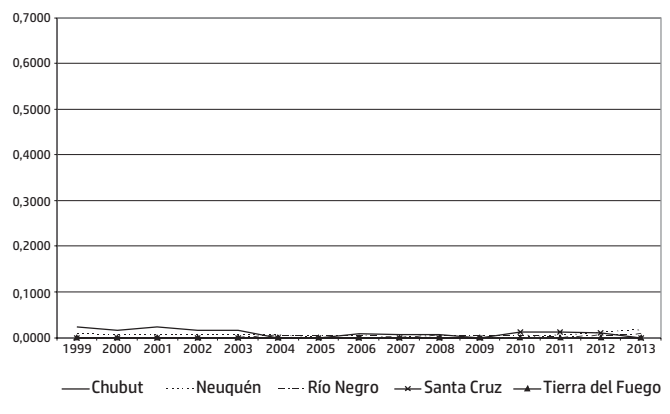

Figura 4. Tasa de mortalidad en la niñez por desnutrición y otras anemias nutricionales en menores de cinco años. Provincias según regiones argentinas, 1999-2013.

Fuente: Elaboración propia con base en datos del Programa Nacional de Estadísticas de Salud, Dirección de Estadísticas e Información de Salud, Ministerio de Salud de la Nación.

mortalidad cambia notoriamente. En primer lugar, se destaca que las provincias con registros muy bajos de muerte por desnutrición no presentan una situación idéntica con relación a los egresos. Nótese, por ejemplo, las elevadas tasas en las provincias de la región pampeana, patagónica e incluso en el Área Metropolitana de Buenos Aires, aunque comparativamente más bajas que las de la región nordeste y noroeste. Esta evidencia pone de manifiesto la severidad del problema que, según la fuente utilizada, si bien no provoca la muerte, constituye un importante problema de salud pública.

Al igual que en el análisis precedente, los valores más elevados están localizados en las provincias de la región noroeste y nordeste, con una preocupante tendencia sostenida al aumento de la tasa salteña. Otro caso que detiene la atención -tanto por su 
Cuyo

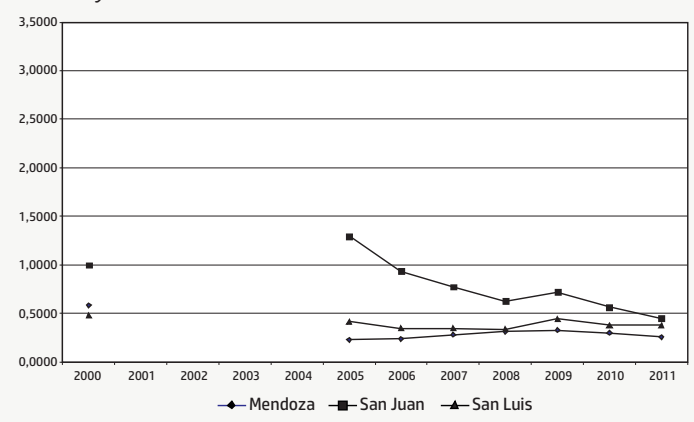

Noroeste

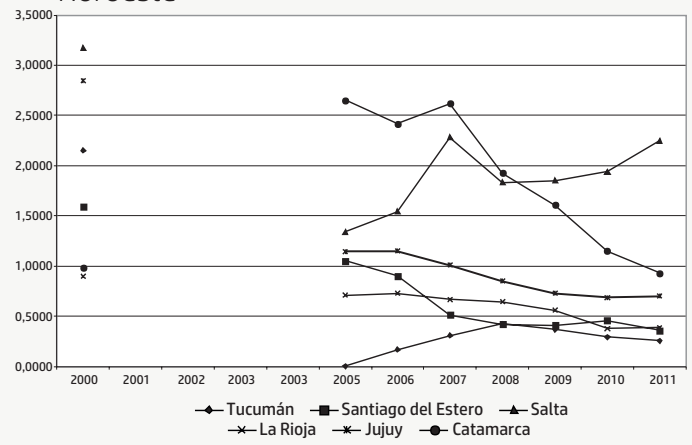

Pampeana

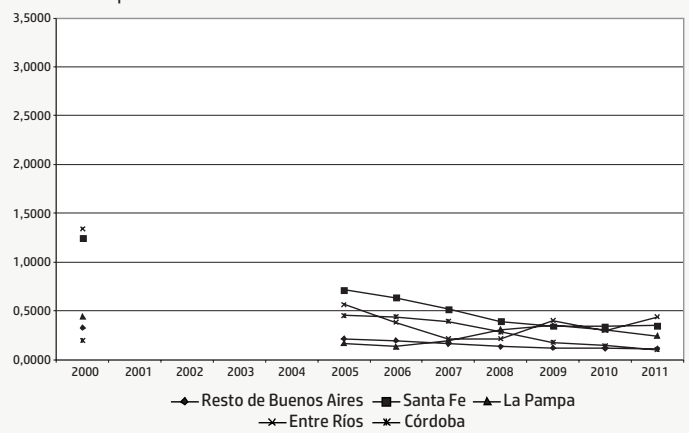

Metropolitana

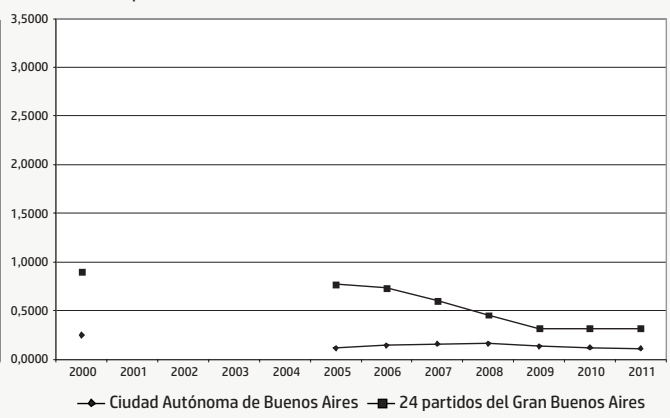

Nordeste

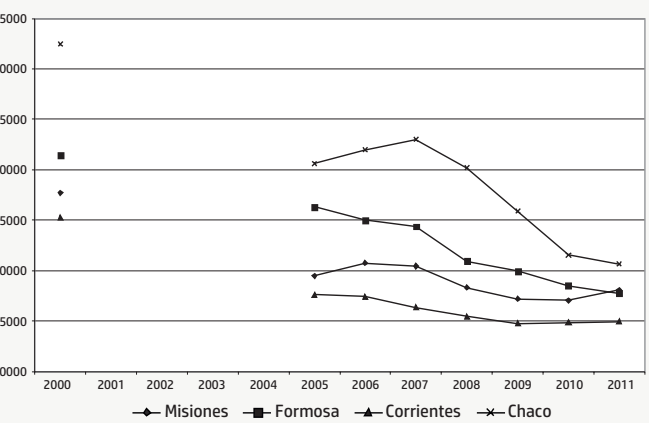

Patagonia

Figura 5. Tasa de egresos hospitalarios por desnutrición y otras anemias nutricionales en menores de cinco años. Provincias según regiones argentinas, 2000-2011.

Fuente: Elaboración propia con base en datos del Programa Nacional de Estadísticas de Salud, Dirección de Estadísticas e Información de Salud, Ministerio de Salud de la Nación.

magnitud como por su tendencia- corresponde a Neuquén, provincia que mostró una importante brecha respecto del resto de las provincias patagónicas, con tasas que superaron el 1,5 por mil en 2009-2010, y un valor absoluto de 199 egresos de menores por esta causa en aquellos años.

El análisis provincial del bajo peso puede observarse en la Figura 6. En este caso, el dominio de la tendencia descendente se ve interrumpido, dominando cierta estabilidad en el indicador, con excepción de las provincias de la región del nordeste. Estas últimas presentan, además, las magnitudes más elevadas, y se destaca la situación de Misiones, que alcanzó en 2007 el 1,6\% de los nacidos vivos bajo las características señaladas. Pusimos el acento sobre tres casos: Tucumán, la 
Cuyo

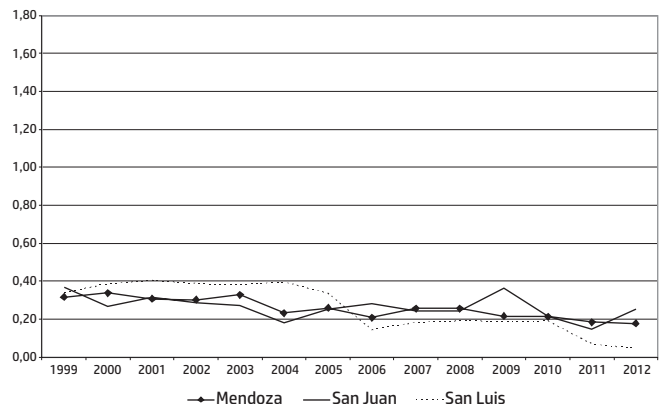

Noroeste

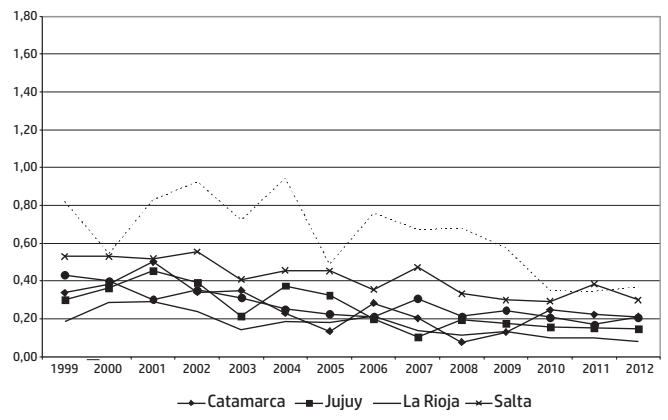

Pampeana

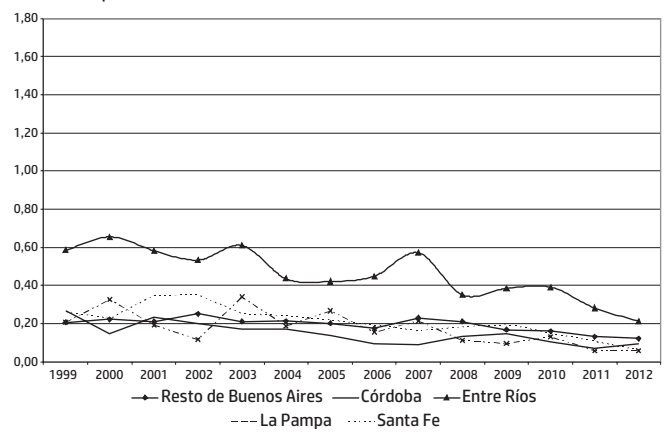

Metropolitana

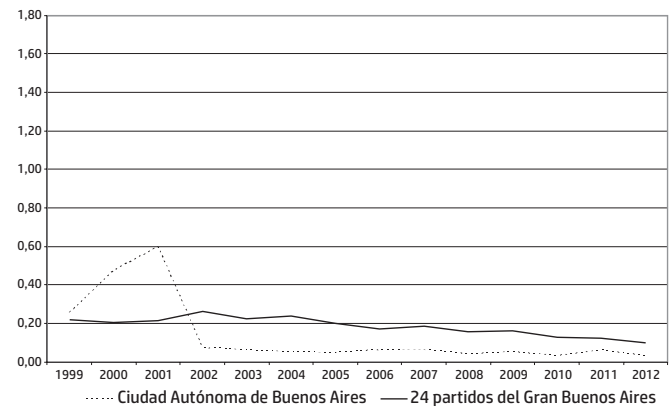

Nordeste

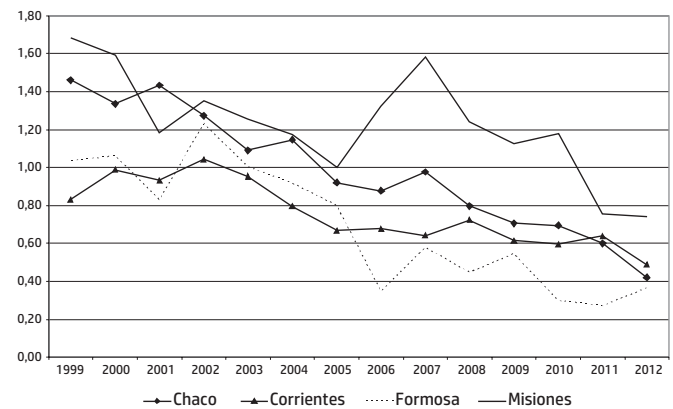

Patagonia

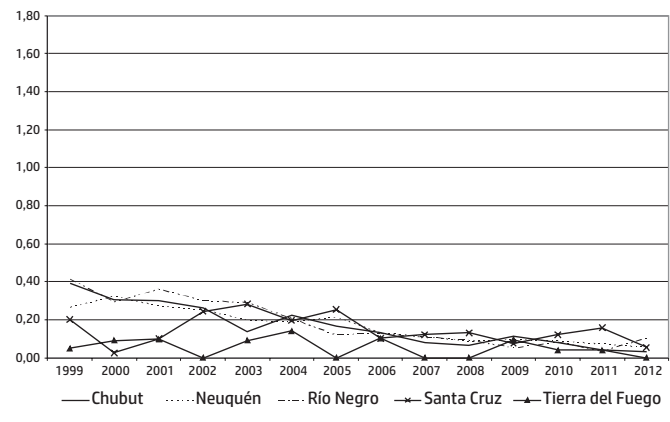

Figura 6. Proporción de nacimientos con bajo peso a término provenientes de madres con educación crítica. Provincias según regiones argentinas, 1999-2012.

Fuente: Elaboración propia con base en datos del Programa Nacional de Estadísticas de Salud, Dirección de Estadísticas e Información de Salud, Ministerio de Salud de la Nación.

Ciudad Autónoma de Buenos Aires y el Área Metropolitana de Buenos Aires. Tucumán tuvo ciertas particularidades en el análisis, ya que mientras las curvas de mortalidad y de egresos hospitalarios son bajas (las de menor magnitud de la región) y descendentes -en el caso de la mortalidad- o estables -en los egresos-, el bajo peso muestra magnitudes más elevadas con un comportamiento casi sin variaciones durante todo el periodo, con valores promedio solo por debajo de Salta y Santiago del Estero. Esta evidencia genera sospecha respecto de la calidad de los datos que dan cuenta de la magnitud del problema, el cual no habría sido captado de manera adecuada a partir de las estadísticas vitales y las de egresos hospitalarios; sospecha que, además, encuentra sustento en los relatos de 

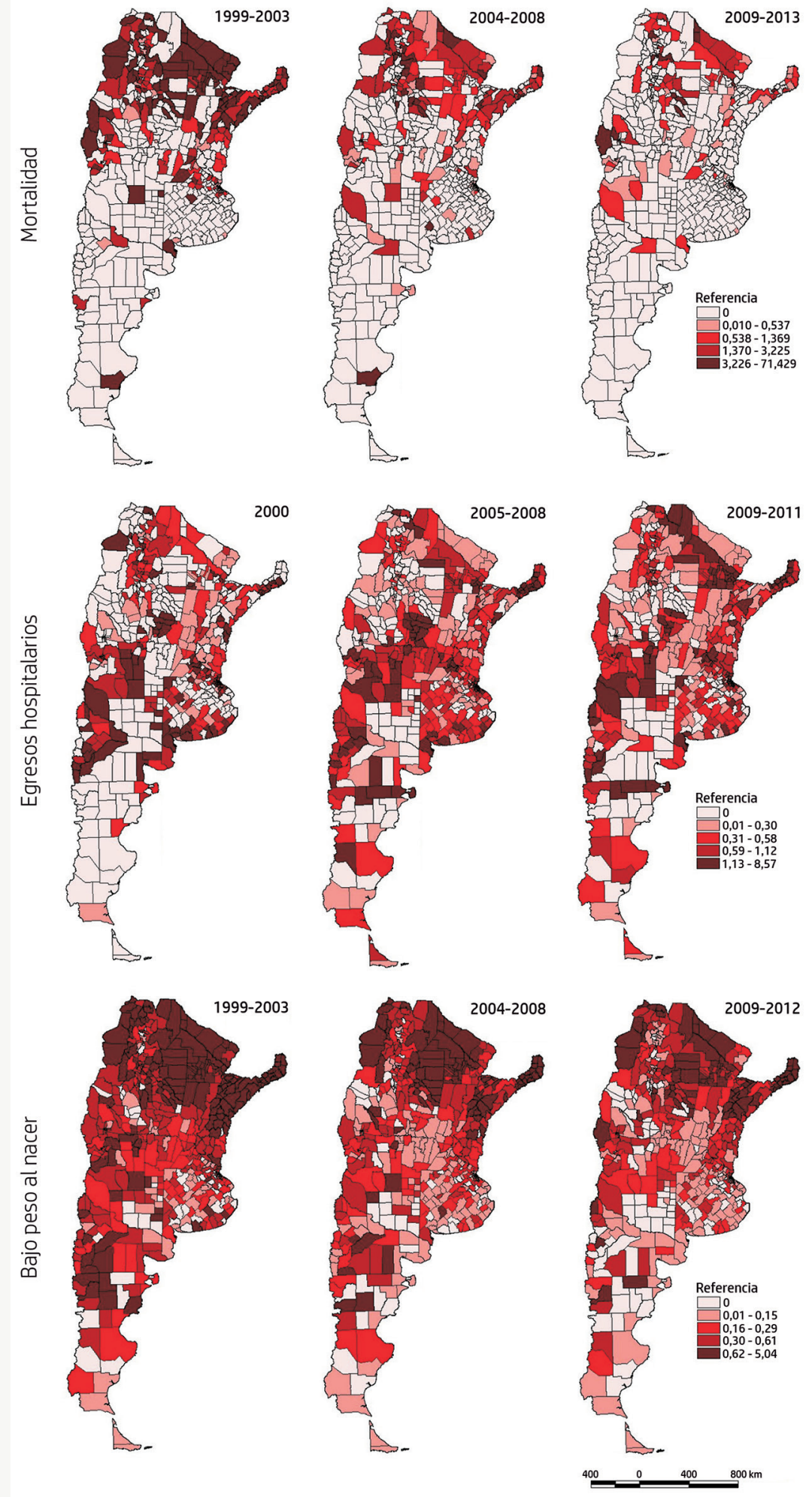

Figura 7. Proporción de muertes y egresos hospitalarios de menores de cinco años bajo la causa "desnutrición y otras anemias nutricionales" según departamentos, y proporción de nacimientos de bajo peso. Argentina, 1999-2013.

Fuente: Elaboración propia con base en datos del Programa Nacional de Estadisticas de Salud, Dirección de Estadísticas e Información de Salud, Ministerio de Salud de la Nación. 
diferentes entrevistados quienes mencionan la criticidad del problema en la provincia y los problemas para captar la información en forma objetiva y fidedigna. Las entrevistas que dan lugar a estas conjeturas formaron parte de una investigación más amplia, pero no fueron analizadas en este artículo. Su mención solo responde a explicar la contradicción que se manifiesta a partir de las fuentes cuantitativas.

En el caso de la Ciudad Autónoma de Buenos Aires y su área metropolitana observamos valores muy bajos de mortalidad, bajos y descendentes en los egresos y bajos y estables en el bajo peso al nacer. Esta condición presenta menos contradicción que Tucumán, donde es posible advertir la presencia de un problema -en cierta medida atendido- con escasos casos que culminan en un desenlace fatal.

\section{La escala departamental}

En la Figura 7, se observa la proporción de muertes/egresos por desnutrición y de bajo peso al nacer en la escala departamental. El registro cartográfico permitió advertir la concentración del problema en vastas regiones del norte argentino. En el caso de la mortalidad, se distingue que, a pesar de la tendencia al descenso que presentó el problema, regiones como el Impenetrable chaco-formoseño y su área oriental, los valles salto-jujeños, el área del interfluvio DulceSalado de Santiago del Estero o algunos departamentos de la meseta misionera como núcleos de elevada magnitud y persistencia de la desnutrición en el norte. A ellos se agregan algunos departamentos sanjuaninos en idéntica condición, que evidencian el aumento de la tasa provincial expuesto en el análisis por provincias.

Los egresos hospitalarios, por otro lado, muestran una concentración del problema que no solo responde a las provincias norteñas. En efecto, adquieren una situación crítica algunos departamentos patagónicos (particularmente, de las provincias de Río Negro, Chubut y Neuquén), y del área que circunda la capital sanjuanina, entre otros. Es evidente que la tendencia al descenso observada en la mortalidad no se presenta con tanta claridad en esta variable, como pudo observarse también en la Figura 2.

Finalmente, el análisis del bajo peso llama la atención por su persistencia extendida en el norte argentino y coincide, en muchos casos, con las áreas señaladas en los casos anteriores. Si bien se observa una reducción del problema, se destacan núcleos que, en los tres quinquenios analizados, se ubicaron en el cuarto cuartil. Estos se corresponden con la Puna jujeña, las comunidades indígenas chaqueñas, el campesinado santiagueño, el chaco algodonero, el campesinado y las comunidades indígenas de la meseta misionera, todos núcleos duros de pobreza identificados por Bolsi y Paolasso ${ }^{(22)}$. Se destaca también el descenso en la concentración del problema en un amplio sector de la Patagonia norte, como así también en numerosos departamentos cuyanos.

Ante los resultados hallados nos preguntamos ¿cómo evolucionó la desnutrición infantil en Argentina?, ¿qué magnitudes presenta actualmente?, ¿cuáles fueron las tendencias regionales y provinciales dominantes?, y ¿cómo evolucionó su distribución espacial?

Los indicadores construidos pudieron detectar que, en el periodo que va de 1999 a 2013, a pesar de grandes avances en materia de reducción de la pobreza y mejora de las condiciones de vida en la población infantil -notorios si se los compara con la década de 1990-, se destaca un importante descenso de la mortalidad por desnutrición, aunque persisten magnitudes elevadas con relación a la morbilidad y el bajo peso. Si bien a nivel nacional el problema ha sido mitigado, puede observarse que, cuando se cambia la escala, algunas regiones duplican los registros iniciales y, en su interior, se encuentran provincias que mostraron estancamiento o incluso un incremento de la tasa. El análisis del bajo peso al nacer evidenció idéntico proceso. Finalmente, el examen cartográfico puso de manifiesto la persistencia y gravedad del problema en distintos sectores del norte argentino, detectando áreas que 
deberían tener urgente intervención. Los departamentos Candelaria (Misiones) y Ullum (San Juan) por ejemplo, presentaron proporciones del $15 \%$ y el $12 \%$ respectivamente de muertes de menores de cinco años por sobre el total de defunciones en este tramo de edad.

\section{DISCUSIONES}

El estudio de las magnitudes de la desnutrición infantil, en general, y de su distribución espacial, en particular, no ha sido profundamente estudiado en nuestro país, de allí que sea difícil contrastar los resultados que aquí se presentan. Se destacan, sin embargo, importantes avances bajo este enfoque en países como Perú, México, Ecuador y República Dominicana ${ }^{(21,23,24,25,26)}$.

No obstante, es preciso remarcar algunos hitos importantes en el estudio territorial de la desnutrición en la niñez, centrando el análisis en el caso argentino. Uno de ellos lo constituye el aporte de Cetrángolo ${ }^{(27)}$ con su denominado "Mapa nutricional argentino", el cual constituye el primer abordaje, de carácter nacional, sobre la distribución espacial de los problemas nutricionales. Durante la década de 1970, Escudero et al. ${ }^{(28)}$ sostenían que, a pesar de que el promedio de calorías consumidas per cápita era óptimo, la existencia de desnutrición era significativa en Argentina. Evidentemente, el problema se concentraba, según afirmaban, en la inequidad del reparto. A pesar del elevado subregistro, detectaron 924 defunciones ocurridas en 1969, específicamente por desnutrición en Argentina.

Las últimas décadas del siglo $\mathrm{XX}$ y los primeros años del siglo XXI vieron proliferar notoriamente trabajos de investigación que ponían énfasis en las secuelas del subconsumo alimentario en los niveles cognitivos, a lo que muchos llamaron el "daño cerebral infantil". Durante el año 2002, el problema de la desnutrición en la niñez argentina tomó una repercusión inusitada, concentrado -originalmente- en la provincia de Tucumán. Fueron numerosos los casos de desnutrición que salieron a la luz y que permitían un "redescubrimiento" del problema, los cuales fueron divulgados en notas de periódicos nacionales e internacionales ${ }^{(29)}$.

En los inicios del siglo XXI, a partir de la realización de la Encuesta Nacional de Salud y Nutrición (2004-2005), se detectaban cifras alarmantes de la situación nutricional infantil en el país: se observó el 8,0\% de acortamiento, el $1,3 \%$ de emaciación y el $10,4 \%$ de obesidad; la prevalencia de anemia fue del $16,5 \%$ en menores de 6 años y del 35,3\% en niños de 6 a 23 meses. Asimismo, se revelaba que los niños que pertenecían a hogares de bajo nivel socioeconómico presentaron mayor prevalencia de condiciones de inadecuación nutricional ${ }^{(7)}$. Podía observarse que el problema de la desnutrición estaba lejos de ser solucionado y adquiría, en los albores del siglo XXI, magnitudes increíbles para Argentina. No obstante, más allá del conocimiento logrado sobre el problema, poco se conoce en la actualidad respecto de la distribución espacial del problema, su magnitud y sus variaciones (regionales, provinciales y departamentales); es decir, dónde se concentra este problema (ya sea tomando la vertiente de mortalidad, morbilidad o bajo peso al nacer) y dónde se intensifica, persiste o se atenúa; qué provincias concentran las mayores proporciones y qué territorios podrían definirse como núcleos duros de desnutrición.

Por los motivos reseñados, este artículo procuró constituir un primer abordaje nacional del problema, con las limitaciones que conlleva constituir un producto de vanguardia en el análisis espacial del problema, entre ellas, las dificultades propias para contrastar los resultados que aquí se presentan.

\section{CONSIDERACIONES FINALES}

La desnutrición infantil conforma el principal núcleo duro de privación y exclusión que debería ser prioritario en las políticas públicas. Existen muchas barreras para su conocimiento y análisis, que dependen, a su vez, 
de las escalas que se utilizan y las fuentes que se emplean.

Luego de los análisis precedentes, queda claro que cada una de las vertientes que se analizan resultan insuficientes para caracterizar el problema de la desnutrición en la niñez, pero el análisis conjunto permite obtener una caracterización y una comprensión del fenómeno mucho más clara. En este sentido, esta investigación pretende sentar las bases para una nueva forma de enfocar el problema de la desnutrición, la cual no se agota en los aspectos aquí estudiados, pero que abre un fértil campo para estudios posteriores sobre la materia.

De una manera casi concluyente puede verse, luego de analizar el problema, que lejos de ser una cuestión del pasado, la desnutrición en la niñez constituye uno de los problemas de mayor relevancia en la actualidad en materia de salud pública. Las evidencias halladas muestran que la desnutrición, a pesar de haber descendido, presenta magnitudes que la posicionan como uno de los más importantes problemas de salud pública en algunas regiones de Argentina, influyendo en gran medida en las altas tasas de morbilidad y mortalidad infantil, así como en la prevalencia de ciertas enfermedades crónicas, difíciles de cuantificar. Asimismo, el costo económico y social de la enfermedad es muy alto también (gastos hospitalarios, productividad, gastos en educación, menor capacidad laboral, etc.), y el daño cerebral irreversible que genera, también complejo de cuantificar, aunque con profundas connotaciones éticas y morales.

Por estos motivos, se insiste sobre la necesidad de continuar con investigaciones que profundicen la aplicación de las herramientas metodológicas aquí presentadas en áreas seleccionadas como estudio de caso, que integren las distintas vertientes para el estudio de la desnutrición que aquí fueron presentadas. Dicha selección de casos operaría sobre la base de la cartografía expuesta, que permitiría brindar conocimiento sobre las características del problema, su avance, retroceso o persistencia y la concatenación de factores a los cuales estaría asociado.

\section{AGRADECIMIENTOS}

Los autores desean agradecer a la Comisión Nacional Salud Investiga (Ministerio de Salud de la Nación), por el apoyo a través del Programa de Becas de Investigación "Ramón Carrillo-Arturo Oñativia", categoría "Estudio multicéntrico", otorgado a la investigación titulada "La desnutrición en la niñez argentina en los primeros años del siglo XXI" (Reg. IS000917 RENIS) que operó bajo el amparo del dictamen ético emitido por la Escuela Latinoamericana de Bioética (La Plata) y de la cual surge el presente artículo. La coordinación de este estudio estuvo a cargo del doctor Fernando Longhi.

\section{REFERENCIAS BIBLIOGRÁFICAS}

1. Mercer R. Salud y pobreza en la Argentina: dime cómo ha sido tu cuna y te diré cómo serás. Voces en el Fénix. 2013;(22):88-97.

2. Servicio de Planificación, Estimación y Evaluación de la Nutrición, Organización de las Naciones Unidas para la Agricultura y la Alimentación. Perfiles nutricionales por países: Argentina [Internet]. Roma: FAO; 2001 [citado 1 oct 2016]. Disponible en: https://tinyurl.com/yb64tnzs.

3. Organización de las Naciones Unidas para la Alimentación y la Agricultura. Panorama de la inseguridad alimentaria en América Latina y el Caribe 
[Internet]. Roma: FAO; 2015 [citado 1 oct 2016]. Disponible en: http://www.fao.org/3/a-i4636s.pdf

4. Britos S. Crisis 2001-2002: pobreza, precios y alimentos. Buenos Aires: Centro de Estudios sobre Nutrición Infantil; 2003.

5. Calvo E, Aguirre P. Crisis de la seguridad alimentaria en la Argentina y estado nutricional en una población vulnerable. Archivos Argentinos de Pediatría. 2005;103(1):71-90.

6. Longhi F. Magnitudes y tendencias de la desnutrición en la niñez argentina durante la primera década del siglo XXI. Población y Salud en Mesoamérica. 2015;13(1):00-00. doi: 10.15517/psm. v13i1.19496.

7. Durán P, Mangialavori G, Biglieri A, Kogan L, Abeyá Gilardon EO. Estudio descriptivo de la situación nutricional en niños de 6-72 meses de la República Argentina: resultados de la Encuesta Nacional de Nutrición y Salud (ENNyS). Archivos Argentinos de Pediatría. 2009;107(5):397-404.

8. Wanden-Berghe C, Camilo M, Culebras J. Conceptos y definiciones de la desnutrición iberoamericana. Nutrición Hospitalaria. 2010;25(Supl 3):1-9.

9. Macías A, Quintero M, Camacho E, Sánchez J. La tridimensionalidad del concepto de nutrición: su relación con la educación para la salud. Revista Chilena de Nutrición. 2009;36(4):1129-1135.

10. Oyhenart E, Dahinten S, Alba JA, Alfaro EL, Bejarano IF, et al. Estado nutricional infanto-juvenil en seis provincias de argentina: variación regional. Revista Argentina de Antropología Biológica. 2008;10(1):1-62.

11. Fondo de las Naciones Unidas para la Infancia. Estado mundial de la infancia [Internet]. Ginebra: UNICEF; 1998 [citado 1 oct 2016]. Disponible en: https://tinyurl.com/y94h8rca.

12. Torrado S. Salud-enfermedad en el primer año de vida, Rosario (1981-1982). Buenos Aires: Centro de Estudios Urbanos y Regionales; 1986.

13. Bronfman M. Como se vive se muere. México: Centro Regional de Investigaciones Multidisciplinarias; 2001.

14. Maluccio JA, Hoddinott J, Behrman JR, Martorell R, Quisumbing AR, Stein AD. The impact of improving nutrition during early childhood on education among Guatemalan adults. The Economic Journal. 2009;119(537):734-763.

15. Martorell R. Efectos de la desnutrición en la salud y desarrollo humano y estrategias efectivas para su prevención. Salud Pública de México. 2007;49(Ed Esp 1):151-151.

16. Hoddinott J, Behrman JR, Maluccio JA, Melgar P, Quisumbing AR, Ramirez-Zea M, Stein AD, Yount KM, Martorell R. Adult consequences of growth failure in early childhood. The American Journal of Clinical Nutrition. 2013;98(5):1170-1178.

17. Martínez R, Fernández A. Desnutrición infantil en América Latina y el Caribe. Desafíos. 2006;(2):4-9.

18. Rice AL, Sacco L, Hyder A, Black RE. Malnutrition and childhood deaths from infectious diseases in developing countries. Bulletin of the World Health Organization. 2000;78(10):12071221.

19. Moreno M. La pobreza: una medición en busca de su contenido conceptual. Santa Rosa: III Jornadas Argentinas de Estudios de Población; Santa Rosa, La Pampa, Argentina, 11-13 oct 1995.

20. O’Donnell A, Carmuega E. La transición epidemiológica y la situación nutricional de nuestros niños. Boletín CESNI. 1998;6:1-24.

21. Sanghvi T, Murray J. Improving child health through nutrition: the nutrition minimum package. Arlington: Partnership for Child Health Care, Basic Support for Institutionalizing Child Survival; 1997.

22. Bolsi A, Paolasso P. (Comp.). Geografía de la pobreza en el Norte Grande Argentino. San Miguel de Tucumán: PNUD, CONICET, UNT; 2009.

23. Perú, Programa Mundial de Alimentos, Naciones Unidas. Mapa de vulnerabilidad a la desnutrición crónica infantil en Perú [Internet]. Lima: Programa Mundial de Alimentos; 2007 [citado 1 oct 2016]. Disponible en: https://tinyurl.com/ y8t9kom7.

24. Ayala-Gaytán EA, Díaz Durán-Hernández A. Infraestructura, ingreso y desnutrición infantil en México. Salud Pública de México. 2015;57(1): 22-28.

25. Dirección de Análisis e Investigación del Ministerio de Coordinación de Desarrollo Social. Mapa de la Desnutrición Crónica en el Ecuador [Internet]. Quito: Programa Mundial de Alimentos; 2010 [citado 1 oct 2016]. Disponible en: http:// mapadesnutricion.org/.

26. Rogers $\mathrm{BL}$, Macías KE, Wilde $\mathrm{P}$. Atlas del hambre y la desnutrición en la República Dominicana [Internet]. Santo Domingo: Programa Mundial de Alimentos; 2007 [citado 1 oct 2016]. Disponible en: https://tinyurl.com/y8zuhbs7. 
27. Cetrángolo HA. Análisis sobre la nutrición en la República Argentina. Prensa Universitaria. 1966;158:2388-2390.

28. Escudero J, Scarponi F, Kotliar H. Un aporte al conocimiento de la desnutrición en la Argentina. Cuadernos de Salud Pública. 1972;8:11-16.
29. Longhi F. Desnutrición y muerte en la niñez argentina en los albores del siglo XXI: un análisis espacial. Journal of Latin American Geography. 2014;13(2):41-65.

\section{FORMA DE CITAR}

Longhi F, Gómez A, Zapata ME, Paolasso P, Olmos F, Ramos Margarido S. La desnutrición en la niñez argentina en los primeros años del siglo XXI: un abordaje cuantitativo. Salud Colectiva. 2018;14(1):33-50. doi: 10.18294/sc.2018.1176

Recibido: 11 de octubre de 2016 | Versión final: 26 de abril de 2017 | Aprobado: 12 de junio de 2017

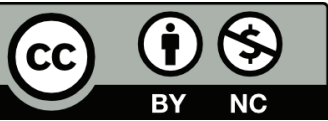

Este obra está bajo una licencia de Creative Commons Reconocimiento-NoComercial 4.0

Internacional. Reconocimiento - Permite copiar, distribuir y comunicar públicamente la obra.

A cambio, se debe reconocer y citar al autor original. No Comercial - Esta obra no puede ser

utilizada con finalidades comerciales, a menos que se obtenga el permiso.

http://dx.doi.org/10.18294/sc.2018.1176 